\title{
Bioactivity of Plectranthus grandis (L.H. Cramer) R.H. Willemse Extracts on Biomphalaria glabrata (Say, 1818) in Laboratory
}

\author{
José Augusto Albuquerque Santos ${ }^{1 *}$, Keyla Nunes Farias Gomes ${ }^{2}$, Lucas Vinicius Lima ${ }^{3}$ and Robson \\ Xavier Faria ${ }^{1}$ \\ ${ }^{1}$ Laboratory of Environmental Health Assessment and Promotion, Oswaldo Cruz Institute, Brasil \\ ${ }^{2}$ Master's degree in Biological Sciences and Biotechnology, Universidade Federal Fluminense, Brasil \\ 3 Joaquim Venancio Polytechnic School of Health, Brasil
}

\begin{abstract}
The objective of this work was to test the bioactivity of the different extracts and fractions of the leaves of Plectranthus grandis and to determine their lethal concentrations on Biomphalaria glabrata. For the bioassays, seven crude extracts obtained in soxhlet extractor with organic solvents and five fractions were used by flash filtration chromatography. The bioassays were performed in $200 \mathrm{~mL}$ glass and 24-well plates, using 1674 mollusks in the bioassays. The results showed that the $500 \mathrm{mg} / \mathrm{L}$ beaker tests obtained $100 \%$ mortality rate for the crude extracts in hexane and dichloromethane and $93 \%$ for chloroform extract. In plaque tests, the dichloromethane extracts the lowest $\mathrm{CL}_{90}=318 \mathrm{mg} / \mathrm{L}$. The mortality rate with the fractions was $100 \%$ at a concentration of $300 \mathrm{mg} / \mathrm{L}$. It was concluded that the tests performed on cups and plates showed significant results.
\end{abstract}

KEYWORDS: Schistosomíasis; Plectranthus grandis; Natural products

\section{INTRODUCTION}

Schistosomiasis is a neglected tropical disease caused by parasitic worms of Schistosoma genus. It is transmitted by intermediate hosts Biomphalaria spp. This disease can impair children's ability to learn and cause anemia and rickets in children, becoming one of the major public health problems [1,2]. According to World Health Organization data, it suggests that over 200 million people have been infected in 78 countries, including 52 endemic countries located in Africa, the Middle East, the Caribbean and South America, resulting in about 200,000 deaths per year [2].

The search for more effective molluscicides with less impact on the environment and with lower cost has motivated the search for plants as a source of possible molluscicidal substances [3]. Thus, studies in past decades on plants with molluscicidal activity did not go forward. There are currently several studies that investigate plants with this potential, and yet they present themselves as a better strategy in relation to synthetic products $[4,5]$. Numerous plant species were evaluated for molluscicide activity, and some were recognized for showing activity on molluscs. However, to date, there are no reports in the literature about this activity with Plectranthus grandis (L.H. Cramer) R.H. Willemse. The Plectranthus genus belongs to the Lamiaceae family. This genus includes about three hundred (300) species distributed throughout the tropical and warm regions of the world with the African continent, India, and Australia [6]. Plectranthus genus was being considered as a plant of the future for its economic potential in various sectors of horticulture, floriculture, ethinobotany, traditional medicine and biology [7].

At moment, there is no product based on natural products for the control of schistosomiasis intermediate host molluscs, and there is no mention in the literature about the molluscicidal activity of Plectranthus grandis. Considering the popular use of

Quick Response Code:

Address for correspondence: José Augusto Albuquerque Santos, Laboratory of Environmental Health Assessment and Promotion, Oswaldo Cruz Institute, Brasil

Received: October 05, 2021

Published: October 13, 2021

How to cite this article: José Augusto AS, Keyla Nunes FG, Lucas VL, Robson XF. Bioactivity of Plectranthus grandis (L.H. Cramer) R.H. Willemse Extracts on Biomphalaria glabrata (Say, 1818) in Laboratory. 2021 - 3(5) OAJBS.ID.000333. DOI: 10.38125/OAJBS.000333 
this plant in several countries and in Brazil, it was decided to study the plant extracts of this species as an alternative in the control of intermediate hosts of schistosomiasis.

\section{MATERIALS AND METHODS}

\section{Plant Material}

Plant material collection and processing: Plectranthus grandis (L.H. Cramer) R.H. Willemse was collected in the municipality of Cabo Frio, Rio de Janeiro State, Brazil. The collection was held in 2016 at coordinates S 22 $37^{\circ}$ '26' and W 42으 01 '19'. Dr. Ray Harley of the Royal Botanical Gardens of Kew properly performed identification and classification at the Botanical Garden of Rio de Janeiro, RJ. The identified species was deposited in the Rio de Janeiro Botanical Garden herbarium under the number RB 762521.

Assays: Preparation of extracts and biological tests: The Laboratory for Evaluation and Promotion of Environmental Health of the Oswaldo Cruz Institute - Fiocruz, prepared and performed the tests with extracts and fractions of Plectranthus grandis (L.H. Cramer) R.H. Willemse leaves.

Mollusks: Biomphalaria glabrata, from the Sumidouro neighborhood located in the city of Rio de Janeiro, RJ, with sizes between $10 \mathrm{~mm}-12 \mathrm{~mm}$ in shell diameter. The creation of the molluscs was according to Paraense [8], performed at Lauro Travassos Pavilion/IOC/FIOCRUZ.

Extracts and fractions preparation: Plectranthus grandis leaves were kiln dried and crushed in a blender. A mass of $10 \mathrm{~g}$ of the powder was extracted by exhaustion in a soxhlet extractor with organic solvents, hexane, dichloromethane, chloroform, ethyl acetate, acetone, ethanol, and methanol to obtain the crude extracts. To obtain the fractions, a crude ethanolic extract was prepared with the leaf powder of Plectranthus grandis, as recommended by Kawai et al. [9] and Makino et al. [10]. he obtained extract followed the same procedure described above. $1.0916 \mathrm{~g}$ of the crude extract in ethanol was used for flash filtration chromatography with polar and medium polar solvents to eliminate grease and fat following the usual course, i.e., treatment with hexane, dichloromethane, ethyl acetate, acetone, and ethanol.

High performance liquid chromatography profiling of fractions of ethanol extract of $P$. grandis leaves. The tests to identify the structures of the substances in the plant with the extract and fractions obtained, employed techniques of identification and characterization of the constituents through techniques of chemical and physicochemical reactions of adsorption or partition, in open column and reverse phase-HPLC. liquid) in accordance with the polarity conditions of the material subjected to subsequent purification steps. Samples of ethanol extract fractions and standards were prepared in the Farmanguinhos Technology Platform - FIOCRUZ. Using a given mass of sample, the $1 \mathrm{mg} / \mathrm{L}$ ethanol solvent was added, which was vortexed and ultrasonically washed for $3 \mathrm{~min}$. It was then diluted 1:10 in water and filtered through a $0.22 \mu \mathrm{m}$ PVDF membrane and injected.

Samples were analyzed by high performance liquid chromatography (NEXERA XR LC-20 model, Shimadzu, Kyoto, Japan) with a matrix detector diode (SPD-M20A) and an automatic injector (SIL-20ADXR). The analysis was performed on a Raptor ACR-C18 Column-2.7 $\mu \mathrm{m}$ and id LC 254, internal diameter-2.1mm and the solvents followed system conditions with a flow rate of $0.5 \mathrm{~mL} / \mathrm{min}$. Were used: (A) $0.2 \%$ aqueous formic acid and (B) acetonitrile. High performance liquid chromatography was monitored at $255 \mathrm{~nm}$. Quercetin, rutin and catechin were used as standards. They were purchased from Sigma-Aldrich $₫$ to compare their chromatograms with chromatographic analyzes of fractions, ethanol extract, $P$. grandis leaves. The chromatogram peaks of the fractions were compared by their retention times with the reference standards.

Concentrations: Concentrated solutions were initially prepared for each crude extract and for each fraction obtained. Dissolutions were performed in dimethyl sulfoxide (DMSO) P.A. Aqueous solutions of the extracts and / or fractions were diluted to concentrations: $50 \mathrm{mg} / \mathrm{L}, 100 \mathrm{mg} / \mathrm{L}, 150 \mathrm{mg} / \mathrm{L}, 200 \mathrm{mg} / \mathrm{L}, 300 \mathrm{mg} / \mathrm{L}$, $400 \mathrm{mg} / \mathrm{L}$ and $500 \mathrm{mg} / \mathrm{L}, 600 \mathrm{mg} / \mathrm{L}, 700 \mathrm{mg} / \mathrm{L}, 800 \mathrm{mg} / \mathrm{L}, 900 \mathrm{mg} / \mathrm{L}$ and $1000 \mathrm{mg} / \mathrm{L}$.

\section{Bioassays}

First bioassay-in glasses: The tests were performed in glass with a capacity of $200 \mathrm{~mL}$. For each type of crude extract and for each bioassay, two negative control groups (one containing only the diluent, distilled water and the other with dimethyl sulfoxide, 1\% aqueous solution); a positive control with $2 \mathrm{mg} / \mathrm{L}$ Niclosamide ${ }^{\circledR}$ solution; and the experimental groups (solutions with extracts) were used. Three glasses with 5 snails per cup were used, making a total of 15 animals with a final volume of $200 \mathrm{~mL}$ per cup. During this period, the snails were exposed for 48 hours to the concentrations of the different extracts and were prevented from surfacing after placing a Styrofoam shield. All tests were performed with three repetitions, totaling 150 molluscs per extract.

Second and third bioassays-in 24-well plates: The tests were performed in 24-well plates using the methodology of Santos et al. [11] where $10-12 \mathrm{~mm}$ B. glabrata mollusks were individualized in the wells of the 24-well plate. Were exposed to negative controls (water and 1\% DMSO), positive control (Niclosamida ${ }^{\circledR} 2 \mathrm{mg} / \mathrm{L}$ ) and concentrations of different crude extracts, for a final volume of $2 \mathrm{~mL}$ per well, with $\mathrm{N}=3$ per concentration. In bioassays, the snails were exposed for 48 hours, and all tests were performed with three repetitions, totaling 117 animals per extract.

In the third bioassay, the same procedure above was used to verify the bioactivity of the hexane, dichloromethane, ethyl acetate, acetone, and ethanol fractions of the ethanol extract of $P$. grandis leaves. All tests were performed with three repetitions, totaling 72 animals per fraction.

Identification of dead mollusks in bioassays: After the exposure period of $48 \mathrm{~h}$, the identification of dead mollusks was performed by observing the appearance of the shells, lack of mobility, odors, soft part retraction, hemolymph release and abnormally projected cephalopod mass outside the shell [6].

\section{Statistical Analysis}

Statistical analysis of the results was performed by arithmetic mean \pm standard deviation. The average of the treated groups was compared with the control group by analysis of variance (ANOVA), linear regression analysis using the Graph Pad Prism 4 program. The dose response between deaths was calculated using the Excel equation of the scatterplot. The difference was considered significant when $p<0.05$.

\section{RESULTS AND DISCUSSION}


The genus Plectranthus is being considered as a plant of the future for its economic potential in various sectors of horticulture, floriculture, ethinobotany, traditional medicine and biology [6]. To date, there is no product on the market for natural plant products to control schistosomiasis intermediate host molluscs, considered a neglected disease, and there is no mention in the literature on molluscicidal activity using different types of extracts and fractions from P. grandis in cambate to schistosomiasis, which can be extended to other trematodes of medical and veterinary importance $[12,13]$.

\section{Plant Extracts and Fractions}

The yields obtained from the extracts were: hexane extract= $6.65 \%$; dichloromethane extract $=10.37 \%$; chloroform extract =9.57; ethyl acetate extract $=13.01 ;$ acetone extract $=14.56 \%$; ethanol extract $=52.03 \%$ and methanol extract $=21.15 \%$. The literature reports the major chemical composition of leaf oil for Plectranthus grandis, with presence of $\alpha$-Copaeno, $\beta$-Bourbonene, trans Caryophyllene, Germacrene-D, $\alpha$-Zingiberene, $\beta$-Bisabolene, Isobornyl 2-methylbutyrate and $\Delta$-Cadinene [2]. In the study by Bandeira et al. [14], the composition of the essential oil of four species of the genus Plectranthus was evaluated, being considered the Lamiaceae family one of the richest in essential oils. The authors used dried leaves and extracted by hydrodistillation in Clevenger apparatus and obtained an essential oil yield of $0.09 \%$ for $P$. grandis. In the work developed by the group, we did not obtain the essential oil of the leaves, but we obtained different crude extracts with polar and nonpolar solvents, using the soxhlet extractor until complete exhaustion, because we wanted to obtain a greater amount of extract for the biological tests.

\section{High Performance Fraction Liquid Chromatography Profile}

Sequential flash filtration chromatography of the ethanol extract of $P$. grandis leaves using different organic solvents was employed as a strategy to separate and characterize their chemical constituents. This process allowed an adequate separation of the substances, to facilitate the possible localization of active principles responsible for their activity. High performance liquid chromatography profiles of quercetin, rutin and catechin standards were obtained at $255 \mathrm{~nm}$ (Figure 1).

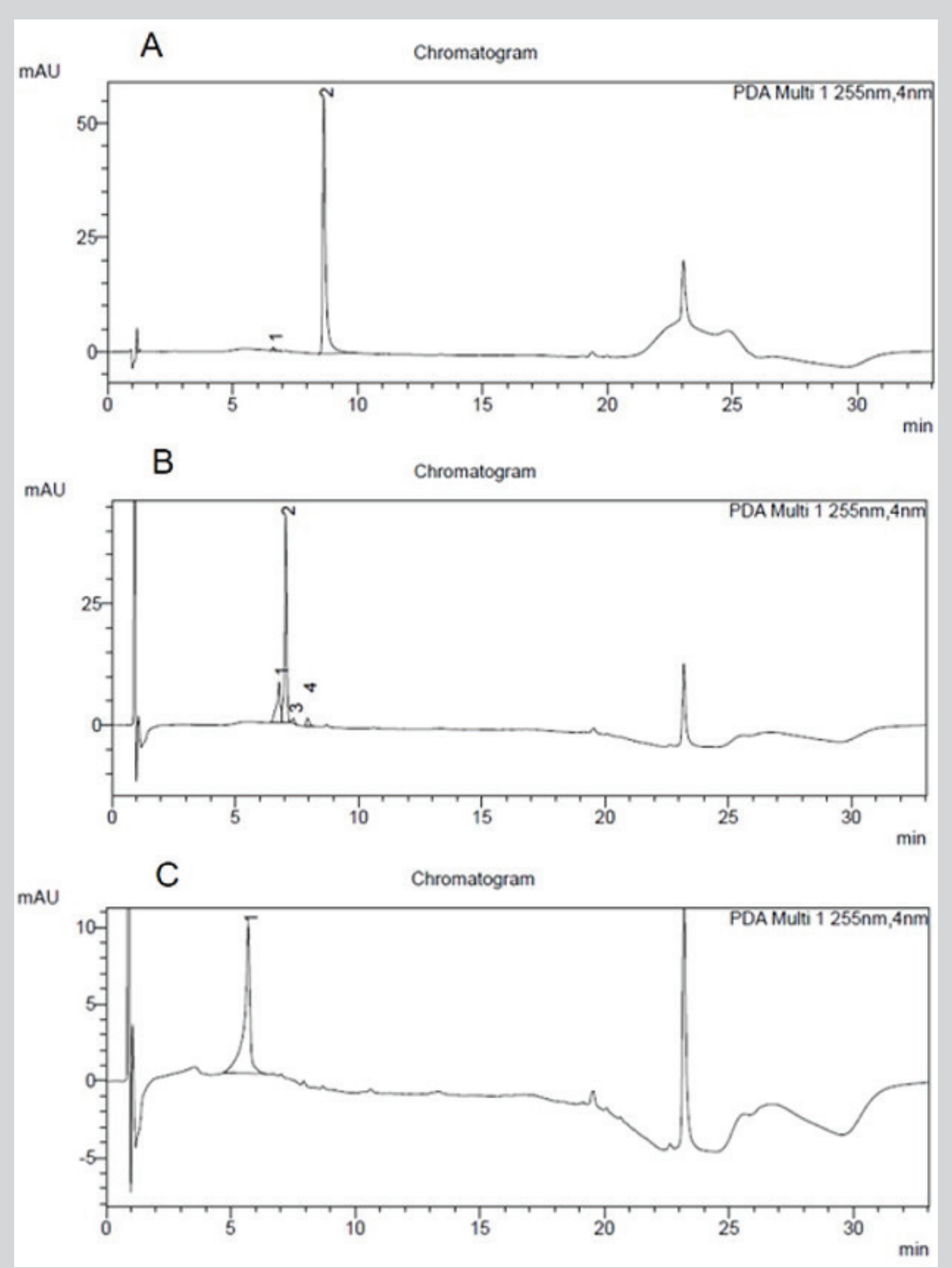

Figure 1: High-performance liquid chromatograms of pattern: A-Quercetin; B-Rutin; C-Catechin. 
The Figure 1 shows the chromatograms of the standards:

(A) quercetin, which showed two peaks in retention times (tR), in minutes of $6.63 \mathrm{~min}$ and $8.66 \mathrm{~min}$, respectively; (B) rutin, which showed four peaks at times $6.78 \mathrm{~min}, 7.05 \mathrm{~min}, 7.36 \mathrm{~min}$ and 7.93min respectively; and (C) catechin, which presented only a retention peak, in the time of $5.71 \mathrm{~min}$.

The results of the chromatographic analysis of the fractions were also obtained at $255 \mathrm{~nm}$ and are shown in Figure 2. The chromatographic analysis showed an important variation in the detected peaks, probably due to a different chemical profile for each fraction. The hexane and acetone fractions of the plant showed in their chromatograms, at peak 4, retention time (tR) of $7.06 \mathrm{~min}$ and at peak 7 , retention time of $6.77 \mathrm{~min}$, with similarities to the rutin standard. The fractions in dichloromethane and ethyl acetate showed retention time at peak $11,6.58 \mathrm{~min}$ and at peak 6 , retention time of 6.62 , respectively, with similarity for quercetin.

The results of the first bioassay showed that at the concentration of $500 \mathrm{mg} / \mathrm{L}$ in a period of 48 hours, there was $100 \%$ mortality for the hexane (Hex) and dichloromethane (DcM) extracts; $93.4 \%$ for the chloroform extract (ECCl3); $20 \%$ for ethyl acetate extract (EAcO); and $13.4 \%$ for the acetone (EAaceto), ethanol (EEtOH) and methanol (EMeOH) extracts (Table 1). Studies on the molluscicidal effect of Cymbopogon winterianus Jowitt (Poaceae) essential oil on Lymnaea columella and Biomphalaria tenagophila was conducted by Costa et al. [15]. In addition, they used the essential oil extracted from fresh leaves using the Clevenger system. The authors used concentrations ranging from $10 \mathrm{mg} / \mathrm{L}$ to $100 \mathrm{mg} / \mathrm{L}$ in the tests and obtained a $100 \%$ mortality rate at the concentration of $80 \mathrm{mg} / \mathrm{L}$ for B. tenagolhila.

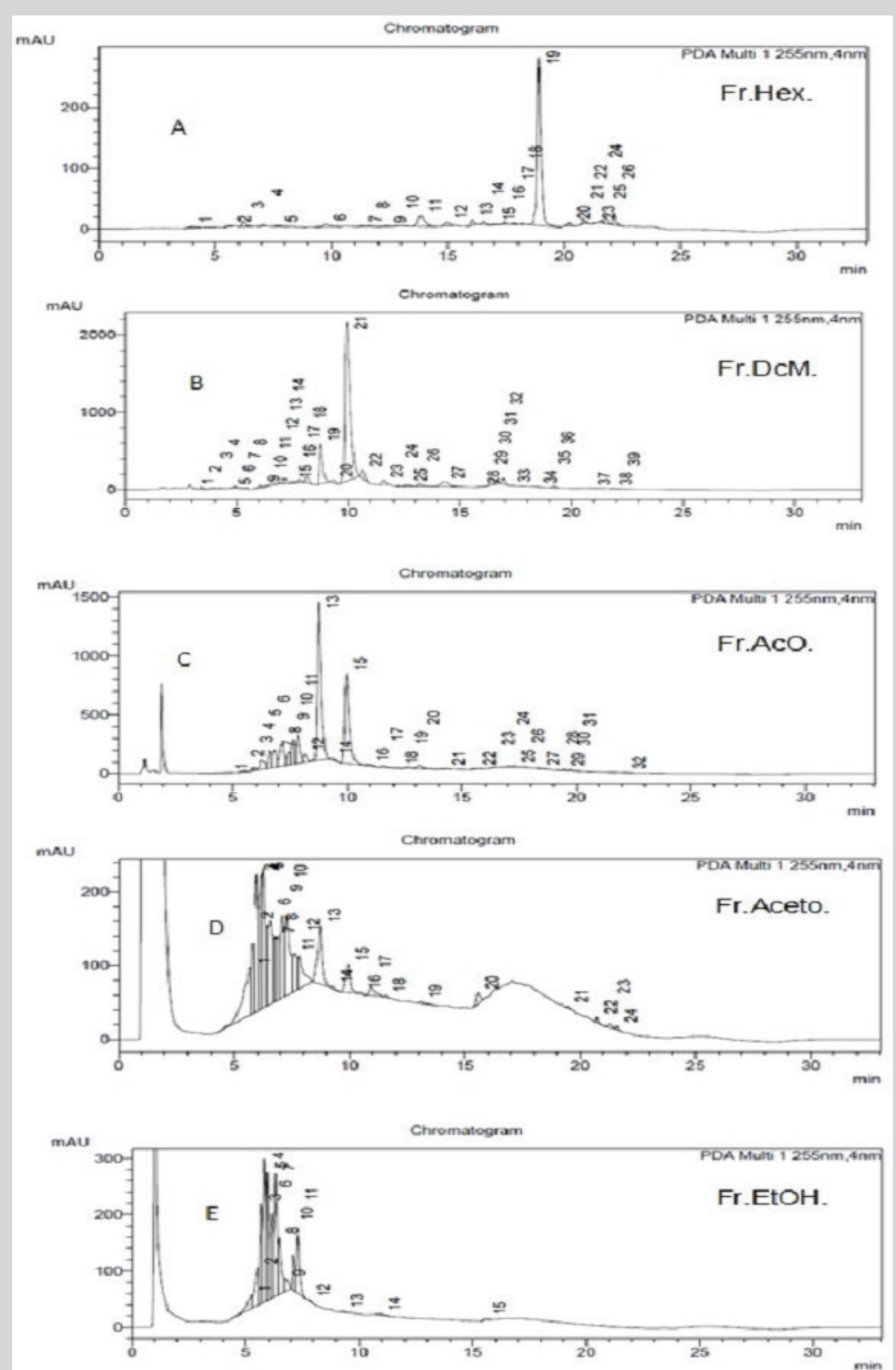

Figure 2: High-performance liquid chromatograms of fractions: A-Hexane fraction; B-Dichoromethane fraction; C-Ethyl acetate fraction; D-Acetone fraction; E-Etanolfraction. 
Table 1: Biomphalaria glabrata Mortality and Lethal Concentration (CL50) in different crude extracts of Plectranthus grandis leaves. First glass bioassay.

\begin{tabular}{|c|c|c|c|c|c|c|c|c|c|c|c|c|c|c|}
\hline \multirow{2}{*}{$\begin{array}{c}\text { Concentrations } \\
(\mathrm{mg} / \mathrm{L})\end{array}$} & \multicolumn{2}{|c|}{ Hex. E. Fo } & \multicolumn{2}{|c|}{ DcM. Fo } & \multicolumn{2}{|c|}{$\mathrm{CCl}_{3}$. E. Fo } & \multicolumn{2}{|c|}{ AcO. E. Fo } & \multicolumn{2}{|c|}{ Aceto. E. Fo } & \multicolumn{2}{|c|}{ EtOH. E. Fo } & \multicolumn{2}{|c|}{ MeOH. E. Fo } \\
\hline & Deaths & $\%$ & Deaths & $\%$ & Deaths & $\%$ & Deaths & $\%$ & Deaths & $\%$ & Deaths & $\%$ & Deaths & $\%$ \\
\hline Water & $0 \pm 0$ & 0 & $0 \pm 0$ & 0 & $0 \pm 0$ & 0 & $0 \pm 0$ & 0 & $0 \pm 0$ & 0 & $0 \pm 0$ & 0 & $0 \pm 0$ & 0 \\
\hline DMSO 1\% & $0 \pm 0$ & 0 & $0 \pm 0$ & 0 & $0 \pm 0$ & 0 & $0 \pm 0$ & 0 & $0 \pm 0$ & 0 & $0 \pm 0$ & 0 & $0 \pm 0$ & 6.6 \\
\hline NCL 2mg/L & $5 \pm 0$ & 100 & $5 \pm 0$ & 100 & $5 \pm 0$ & 100 & $5 \pm 0$ & 100 & $5 \pm 0$ & 100 & $5 \pm 0$ & 100 & $5 \pm 0$ & 100 \\
\hline 50 & $0 \pm 0$ & 0 & $0.33 \pm 0.58$ & 6.6 & $0 \pm 0$ & 0 & $0 \pm 0$ & 0 & $0.33 \pm 0.58$ & 6.6 & $0 \pm 0$ & 0 & $0 \pm 0$ & 0 \\
\hline 100 & $0 \pm 0$ & 0 & $0 \pm 0$ & $0 \pm 0$ & $0 \pm 0$ & 0 & $0.33 \pm 0.58$ & 6.6 & $0 \pm 0$ & 0 & $0 \pm 0$ & 0 & $0 \pm 0$ & 0 \\
\hline 150 & $0 \pm 0$ & 0 & $0 \pm 0$ & $0 \pm 0$ & $0 \pm 0$ & 0 & $0 \pm 0$ & 0 & $0 \pm 0$ & 0 & $0 \pm 0$ & 0 & $0 \pm 0$ & 0 \\
\hline 200 & $0 \pm 0$ & 0 & $1 \pm 0$ & 20 & $0 \pm 0$ & 0 & $0 \pm 0$ & 0 & $0 \pm 0$ & 0 & $0.33 \pm 0.58$ & 6.6 & $0.33 \pm 0.58$ & 6.6 \\
\hline 300 & $0 \pm 0$ & 0 & $0.67 \pm 1.15$ & 13,4 & $1 \pm 0$ & 20 & $2.33 \pm 2.52$ & 46,6 & $0 \pm 0$ & 0 & $0 \pm 0$ & 0 & $0 \pm 0$ & 0 \\
\hline 400 & $0 \pm 0$ & 0 & $3.67 \pm 2.31$ & 73,4 & $3 \pm 2$ & 60 & $1.33 \pm 1.53$ & 26,6 & $1 \pm 0$ & 20 & $0.33 \pm 0.58$ & 6.6 & $0.33 \pm 0.58$ & 6.6 \\
\hline 500 & $5 \pm 0$ & 100 & $5 \pm 0$ & 100 & $4.67 \pm 0.58$ & 93,4 & $1 \pm 0$ & 20 & $0.67 \pm 1.15$ & 13,4 & $0.67 \pm 1.15$ & 13,4 & $0.67 \pm 1.15$ & 13.4 \\
\hline CL50 & \multicolumn{2}{|c|}{339.4} & \multicolumn{2}{|c|}{299.4} & \multicolumn{2}{|c|}{364.4} & \multicolumn{2}{|c|}{498.5} & \multicolumn{2}{|c|}{483.5} & \multicolumn{2}{|c|}{398.5} & \multicolumn{2}{|l|}{0} \\
\hline$P$ value & \multicolumn{2}{|c|}{0.049} & \multicolumn{2}{|c|}{0.0638} & \multicolumn{2}{|c|}{0.0099} & \multicolumn{2}{|c|}{0.0022} & \multicolumn{2}{|c|}{0.0124} & \multicolumn{2}{|c|}{0.0703} & \multicolumn{2}{|c|}{0.06} \\
\hline
\end{tabular}

DMSO: Dimethyl Sulfoxide; NCL: Niclosamide®; SD: Standard Deviation; Hex.E.Fo: Hexane Extract of Leaves, DcM.E.Fo: Dichloromethane Extract of Leaves; $\mathrm{CCl}_{3}$.E.Fo: Chloroform extract of leaves; AcO.E.Fo: Ethyl Acetate Extract of Leaves; Aceto.E.Fo: Acetone Extract of Leaves; E†OH.E.Fo: Ethanol Extract of Leaves; MeOH.E.Fo: Methanol Extract of Leaves. The $\mathrm{CL}_{50}$ were calculated by the Nonlinear Regression Analysis for Dose - Response, using the Graph Pad Prism 5 program, $P=$ Probability, by Linear Regression Analysis, presented $P<0.05$, Significant, $P>0.05$, Not Significant. Test performed with three replicates. Number of animals per concentration ( $N=5)$.

As there is no record in the literature on the use of P. grandis showing biological activity on intermediate hosts of schistosomiasis, we used, in the first moment, glass tests, in concentrations ranging from $50 \mathrm{mg} / \mathrm{L}$ to $500 \mathrm{mg} / \mathrm{L}$, and we obtained $\mathrm{CL}_{50}$ above $299 \mathrm{mg} / \mathrm{L}$ for crude leaf extracts. The results of the Lethal Concentrations $\left(\mathrm{CL}_{50}\right)$ glass tests obtained with the crude leaf extracts of $P$. grandis on $B$. glabrata. It was only possible to determine the $\mathrm{CL}_{50}$ for the glass crude extracts, which was above $300 \mathrm{mg} / \mathrm{L}$, except for the methanol extract that could not calculate the CL (Table 1).

The statistical results of the tests on glasses with crude extracts in hexane, dichloromethane, chloroform, and ethanol from P. grandis leaves on B. glabrata, evaluated by linear regression analysis, showed values of $p=0.0099, p=0.0022, p=0.0124$ and $p$ $=0.049$, respectively and the results are shown in Table 1 . When we performed the linear regression analysis on the tests, we observed that four crude extracts were significant. The work developed by Costa et al. [15], showed that the authors did not perform statistical analysis. Table 2 shows the average mortality of B. glabrata in 24-well plates with different concentrations of $P$. grandis crude extracts performed in the second bioassay. The extract in hexane, with $500 \mathrm{mg} / \mathrm{L}$, presented mortality of $55.67 \%$, and with $600 \mathrm{mg} / \mathrm{L}$ presented mortality of $100 \%$. For the dichloromentane extract, the death rate was $77.7 \%$ with $500 \mathrm{mg} / \mathrm{L}$, and $100 \%$ above this concentration.

For chloroform extract, the initial death rate was $11 \%$ at a concentration of $600 \mathrm{mg} / \mathrm{L}$ and the final rate was $100 \%$ with $800 \mathrm{mg} / \mathrm{L}$. The initial mortality rate found in the ethyl acetate extract was $11 \%$ with $500 \mathrm{mg} / \mathrm{L}$. The acetone extract had an initial death rate of $11 \%$ with $700 \mathrm{mg} / \mathrm{L}$, and the methanol extract had an initial death rate of $22.3 \%$. The ethanol extract showed no mortality.

In plaque tests, the acetone extract had the lowest $\mathrm{CL}_{50}=$ $130 \mathrm{mg} / \mathrm{L}$ and the dichloromethane extract the lowest $\mathrm{CL}_{90}=$ $318 \mathrm{mg} / \mathrm{L}$. For the ethanol extract in plaque, it was not possible to calculate the CLs (Table 2).
The second step involved the use of the 24-well plate test for crude extracts and ethanol extract fractions of the plant leaves. The crude extracts in hexane and dichloromethane of the leaves presented $100 \%$ mortality rate with $600 \mathrm{mg} / \mathrm{L}$, being possible to calculate the $\mathrm{CL}_{50}$ and $\mathrm{CL}_{90}$. In the plaque test, the crude extract with the lowest $\mathrm{CL}_{90}$ was the dichloromethane extract, with $\mathrm{CL}_{90}$ $=318 \mathrm{mg} / \mathrm{L}$. Table 2 highlights the statistical results of 24 -well plate tests with crude extracts of $P$. grandis leaves on B. glabrata by Linear Regression analysis. It shows that the hexane, dichloromethane, and chloroform extracts were significant within $48 \mathrm{~h}$ of exposure.

Table 3 shows the results of the third bioassay, with the average mortality of B. glabrata in 24-well plates with different concentrations of P.grandis leaf fractions. This Table 3 shows that the fractions in hexane, dichloromethane, ethyl acetate and ethanol were significant when analyzed by linear regression analysis. Table 3 shows the results of the plaque tests of Lethal Concentrations $\left(\mathrm{CL}_{50}\right.$ and $\left.\mathrm{CL}_{90}\right)$ obtained from fractions of $P$. grandis leaves on $B$. glabrata.

The fractions with the lowest CLs were the acetone fractions of the leaves, with $\mathrm{CL}_{50}=101.52 \mathrm{mg} / \mathrm{L}$ and the $\mathrm{CL}_{90}=179.96 \mathrm{mg} / \mathrm{L}$, and the ethanol fraction of the leaves, with $\mathrm{CL}_{50}=106.56 \mathrm{mg} / \mathrm{L}$ and $\mathrm{CL}_{90}=188.21 \mathrm{mg} / \mathrm{L}$. With the positive results of bioactivity with the crude extracts, we started to fractionate the crude ethanol extract of the leaves, to evaluate the action of the fractions on B. glabrata in expectation of obtaining lower CL's than those obtained in the crude extracts. Through the tests with the fractions, it was possible to calculate the $\mathrm{CL}_{50}$ and $\mathrm{CL}_{90}$ for each fraction and the ones that presented lower $\mathrm{CL}_{90}$ were the acetone and ethanol fractions of the leaves, with $\mathrm{CL}_{90}=179.96$ and $\mathrm{CL}_{90}=188.21$, respectively.

Our work corroborates those observed by Santos et al. [11] who tested the molluscicidal activity of Physalis angulata extracts and fractions on B. tenagophila and obtained $\mathrm{CL}_{90}=201.2 \mathrm{mg} / \mathrm{L}$ for the leaf physaline pool. Santos et al. [16] used mollusks with a shell diameter of $6-10 \mathrm{~mm}$ to verify the molluscicidal activity of $P$. angulata and $10-12 \mathrm{~mm}$ in the work of a new technique to 
evaluate molluscicidal activity using low volumes. Otarigho [17], studying the molluscicidal effect of Cymbopogon citratus leaves and aqueous extracts against the different stages of development of
Biomphalaria pfeifferi, used adult animals with shell size between 6-8mm, obtaining $\mathrm{CL}_{90}=159.47 \mathrm{mg} / \mathrm{L}$ for ethanolic extract and $\mathrm{CL}_{90}=254.92 \mathrm{mg} / \mathrm{L}$ for aqueous extract.

Table 2: Mortality and Lethal Concentrations for Biomphalaria glabrata in different crude extracts of Plactranthus grandis leaves in 24-well plates.

\begin{tabular}{|c|c|c|c|c|c|c|c|c|c|c|c|c|c|c|}
\hline \multirow{2}{*}{$\begin{array}{c}\text { Concentrations } \\
(\mathrm{mg} / \mathrm{L})\end{array}$} & \multicolumn{2}{|c|}{ Hex. E. Fo } & \multicolumn{2}{|c|}{ DcM. Fo } & \multicolumn{2}{|c|}{$\mathrm{CCl}_{3}$. E. Fo } & \multicolumn{2}{|c|}{ AcO. E. Fo } & \multicolumn{2}{|c|}{ Aceto. E. Fo } & \multicolumn{2}{|c|}{ EtOH. E. Fo } & \multicolumn{2}{|c|}{ MeOH. E. Fo } \\
\hline & Deaths & $\%$ & Deaths & $\%$ & Deaths & $\%$ & Deaths & $\%$ & Deaths & $\%$ & Deaths & $\%$ & Deaths & $\%$ \\
\hline Water & $0 \pm 0$ & 0 & $0 \pm 0$ & 0 & $0 \pm 0$ & 0 & $0 \pm 0$ & 0 & $0 \pm 0$ & 0 & $0 \pm 0$ & 0 & $0 \pm 0$ & 0 \\
\hline DMSO 1\% & $0 \pm 0$ & 0 & $0 \pm 0$ & 0 & $0 \pm 0$ & 0 & $0 \pm 0$ & 0 & $0 \pm 0$ & 0 & $0 \pm 0$ & 0 & $0 \pm 0$ & 0 \\
\hline NCL 2mg/L & $2 \pm 1$ & 67 & $2 \pm 1$ & 67 & $3 \pm 0$ & 100 & $3 \pm 0$ & 100 & $3 \pm 0$ & 100 & $0 \pm 0$ & 0 & $2 \pm 1$ & 67 \\
\hline 100 & $0 \pm 0$ & 0 & $0 \pm 0$ & 0 & $0 \pm 0$ & 0 & $0 \pm 0$ & 0 & $0 \pm 0$ & 0 & $0 \pm 0$ & 0 & $0 \pm 0$ & 0 \\
\hline 200 & $0 \pm 0$ & 0 & $0 \pm 0$ & 0 & $0 \pm 0$ & 0 & $0 \pm 0$ & 0 & $0.33 \pm 0.58$ & 11 & $0 \pm 0$ & 0 & $0 \pm 0$ & 0 \\
\hline 300 & $0 \pm 0$ & 0 & $0 \pm 0$ & 0 & $0 \pm 0$ & 0 & $0 \pm 0$ & 0 & $0 \pm 0$ & 0 & $0 \pm 0$ & 0 & $0 \pm 0$ & 0 \\
\hline 400 & $0 \pm 0$ & 0 & $1 \pm 1$ & 33,3 & $0 \pm 0$ & 0 & $0 \pm 0$ & 0 & $0 \pm 0$ & 0 & $0 \pm 0$ & 0 & $0 \pm 0$ & 0 \\
\hline 500 & $0.67 \pm 0.58$ & 55.67 & $2.33 \pm 0.58$ & 77.7 & $0 \pm 0$ & 0 & $0.33 \pm 0.58$ & 11 & $0 \pm 0$ & 0 & $0 \pm 0$ & 0 & $0 \pm 0$ & 0 \\
\hline 600 & $2 \pm 1$ & 67 & $3 \pm 0$ & 100 & $0.33 \pm 0.58$ & 11 & $0 \pm 0$ & 0 & $0 \pm 0$ & 0 & $0 \pm 0$ & 0 & $0.67 \pm 0.58$ & 22.3 \\
\hline 700 & $3 \pm 0$ & 100 & $3 \pm 0$ & 100 & $2.33 \pm 1.15$ & 77.7 & $0 \pm 0$ & 0 & $0.33 \pm 1.58$ & 11 & $0 \pm 0$ & 0 & $0 \pm 0$ & 0 \\
\hline 800 & $3 \pm 0$ & 100 & $3 \pm 0$ & 100 & $2.67 \pm 0.51$ & 89 & $0 \pm 0$ & 0 & $0 \pm 0$ & 0 & $0 \pm 0$ & 0 & $0 \pm 0$ & 0 \\
\hline 900 & $3 \pm 0$ & 100 & $3 \pm 0$ & 100 & $3 \pm 0$ & 100 & $0.67 \pm 0.58$ & 22.3 & $0.33 \pm 0.58$ & 11 & $0 \pm 0$ & 0 & $0 \pm 0$ & 0 \\
\hline 1000 & $3 \pm 0$ & 100 & $3 \pm 0$ & 100 & $3 \pm 0$ & 100 & $0 \pm 0$ & 0 & $0 \pm 0$ & 0 & $0 \pm 0$ & 0 & $0 \pm 0$ & 0 \\
\hline CL50 & \multicolumn{2}{|c|}{200} & \multicolumn{2}{|c|}{150} & \multicolumn{2}{|c|}{269.04} & \multicolumn{2}{|l|}{185} & \multicolumn{2}{|l|}{130} & \multicolumn{2}{|l|}{0} & \multicolumn{2}{|c|}{216.67} \\
\hline CL90 & \multicolumn{2}{|c|}{392} & \multicolumn{2}{|c|}{318} & \multicolumn{2}{|c|}{457.61} & \multicolumn{2}{|l|}{413} & \multicolumn{2}{|l|}{514} & \multicolumn{2}{|l|}{0} & \multicolumn{2}{|c|}{576.67} \\
\hline$P$ value & \multicolumn{2}{|c|}{$<0.0001$} & \multicolumn{2}{|c|}{$<0.0001$} & \multicolumn{2}{|c|}{0.0004} & \multicolumn{2}{|c|}{0.2572} & \multicolumn{2}{|c|}{0.5683} & \multicolumn{2}{|l|}{0} & \multicolumn{2}{|c|}{0.7699} \\
\hline
\end{tabular}

DMSO: Dimethyl Sulfoxide; NCL: Niclosamide®; SD: Standard Deviation; Hex.E.Fo: Hexane Extract of Leaves, DcM.E.Fo: Dichloromethane Extract of Leaves; $\mathrm{CCl}_{3}$.E.Fo: Chloroform extract of leaves; AcO.E.Fo: Ethyl Acetate Extract of Leaves; Aceto.E.Fo: Acetone Extract of Leaves; E†OH.E.Fo: Ethanol Extract of Leaves; MeOH.E.Fo: Methanol Extract of Leaves. The $\mathrm{CL}_{50}$ were calculated by the Nonlinear Regression Analysis for Dose - Response, using the Graph Pad Prism 5 program, $P=$ Probability, by Linear Regression Analysis, presented $P<0.05$, Significant, $P>0.05$, Not Significant. Test performed with three replicates. Number of animals per concentration ( $N=5)$.

Table 3: Mortality and Lethal Concentrations for Biomphalaria glabrata in different crude extracts of Plactranthus grandis leaves in 24-well plates.

\begin{tabular}{|c|c|c|c|c|c|c|c|c|c|c|}
\hline \multirow{2}{*}{$\begin{array}{c}\text { Concentrations } \\
(\mathrm{mg} / \mathrm{L})\end{array}$} & \multicolumn{2}{|c|}{ Hex. E. Fo } & \multicolumn{2}{|c|}{ DcM. Fo } & \multicolumn{2}{|c|}{ AcO. E. Fo } & \multicolumn{2}{|c|}{ Aceto. E. Fo } & \multicolumn{2}{|c|}{ EtOH. E. Fo } \\
\hline & Deaths & $\%$ & Deaths & $\%$ & Deaths & $\%$ & Deaths & $\%$ & Deaths & $\%$ \\
\hline Water & $0 \pm 0$ & 0 & $0 \pm 0$ & 0 & $0 \pm 0$ & 0 & $0 \pm 0$ & 0 & $0.67 \pm 0.58$ & 22.33 \\
\hline DMSO 1\% & $3 \pm 0$ & 100 & $3 \pm 0$ & 100 & $3 \pm 0$ & 100 & $3 \pm 0$ & 100 & $3 \pm 0$ & 100 \\
\hline NCL 2mg/L & $0.33 \pm 0.58$ & 11 & $0.33 \pm 0.58$ & 11 & $0.33 \pm 0.58$ & 11 & $0 \pm 0$ & 0 & $0.33 \pm 0.58$ & 11 \\
\hline 50 & $0 \pm 0$ & 0 & $0 \pm 0$ & 0 & $0.33 \pm 0.58$ & 11 & $0 \pm 0$ & 0 & $0 \pm 0$ & 0 \\
\hline 100 & $0 \pm 0$ & 0 & $0.67 \pm 0.58$ & 22.33 & $0.33 \pm 0.58$ & 11 & $0.33 \pm 0.58$ & 11 & $0.67 \pm 1.15$ & 22.33 \\
\hline 150 & $1,33 \pm 1.15$ & 44.33 & $0.67 \pm 0.58$ & 22.33 & $0 \pm 0$ & 0 & $1.67 \pm 1.15$ & 55,7 & $2.67 \pm 0.58$ & 89 \\
\hline 200 & $2,33 \pm 0.58$ & 77.66 & $2.67 \pm 0.58$ & 89 & $2.33 \pm 1.15$ & 77.7 & $3 \pm 0$ & 100 & $3 \pm 0$ & 100 \\
\hline 300 & $3 \pm 0$ & 100 & $3 \pm 0$ & 100 & $1.67 \pm 1.15$ & 55.7 & $3 \pm 0$ & 100 & $3 \pm 0$ & 100 \\
\hline CL50 & \multicolumn{2}{|c|}{167.12} & \multicolumn{2}{|c|}{134.71} & \multicolumn{2}{|c|}{245.34} & \multicolumn{2}{|c|}{101.52} & \multicolumn{2}{|c|}{106.56} \\
\hline CL90 & \multicolumn{2}{|c|}{270.56} & \multicolumn{2}{|c|}{239.97} & \multicolumn{2}{|c|}{432.84} & \multicolumn{2}{|c|}{179.96} & \multicolumn{2}{|c|}{188.21} \\
\hline$P$ value & \multicolumn{2}{|c|}{0.0044} & \multicolumn{2}{|c|}{0.0074} & \multicolumn{2}{|c|}{0.0889} & \multicolumn{2}{|c|}{0.0076} & \multicolumn{2}{|c|}{0.0288} \\
\hline
\end{tabular}

DMSO: Dimethyl Sulfoxide; NCL: Niclosamide®; SD: Standard Deviation; Hex.E.Fo: Hexane Extract of Leaves, DcM.E.Fo: Dichloromethane Extract of Leaves; AcO.Fr.Fo: Ethyl Acetate Fraction of Leaves; Aceto.Fr.Fo: Acetône fraction of leaves; EłOH.Fr.Fo: Ethanol fraction of leaves. Test performed with three replicates. Data of $48 \mathrm{~h}$ of test. Analysis by mean, standard deviation and linear regression. $P=$ Probability, $P>0.05$, not significant and $P<0.05$, significant. The $C L_{50}$ and $C L_{90}, w$, calculated by the equation of the straight line of the scatter plot obtained in the Excel Program. Number of animals per concentration $(\mathrm{N}=3)$. 
The work of Araújo et al. [18] on the use of nanoemulsion containing essential oil from Xylopia ochrantha Mart. producing molluscicidal effects against different species of Biomphalaria, they used the previously obtained $\mathrm{CL}_{25}, \mathrm{CL}_{50}$ and $\mathrm{CL}_{90}$ for B. glabrata to perform the tests with different species and different shell sizes.

In our study, we did not use different species of mollusks and different shell sizes, because as there were no reports in theliterature about the use of $P$. grandis showing activity against schistosomiasis intermediate hosts, we decided to use only B. glabrata species, as more susceptible to infection, as a standardization for activity verification.

These bioactivity results will be followed by further investigations with prospects for studies on other vector species of veterinary interest, their larval and embryonic forms, whose concentration can be enhanced with the use of nanoemulsions and/ or plant fractions.

\section{CONCLUSION}

It can be concluded that with the results obtained with the tests performed in 24 well cups and plates for the 7 types of crude extracts and 5 fractions of P. grandis, this species can be considered as a promising molluscicide for presenting significant results, showing the presence of bioactivity of extracts and fractions of P. grandis leaves in the control of schistosomiasis.

\section{AUTHORS' CONTRIBUTION}

JAAS, LVS, RXF and KNFG performed the biological experiments; JAAS general project coordinator, collected the plant material, took the material for botanical identification; He was responsible for the development of biological experiments, wrote the manuscript and performed the statistical analysis. All authors read and approved the manuscript. The authors declare no conflicts of interest.

\section{ACKNOWLEDGMENT}

Instituto Oswaldo Cruz in providing the necessary conditions to carry out the work. To the National Research Council, CNPq for providing an allowance for the student.

\section{REFERENCES}

1. (1994) WHO World Health Organization. The control of schistosomiasis: Second report of the WHO expert committee. In: Fiocruz (Ed.), Rio de Janeiro, Brazil.

2. World Health Organization (2017) Schistosomiasis.

3. Mott KE (1987) Plant molluscicides. UNDP/World Bank/WHO, John Wiley \& Sons Ltd., New York, USA, p. 326.

4. Rapado LN, Nakano E, Ohlweiler FP, Kato MJ, Yamaguchi LF, et al. (2011) T. Molluscicidal and ovicidal activities of plant extracts of the Piperaceae on Biomphalaria glabrata (Say, 1818). Journal of Helminthology 85(1): 66-72.
5. Rapado LN, Pinheiro AS, Lopes PO, Fokoue HH, Scotti MT, et al. (2013) Schistosomiasis control using piplartine against Biomphalaria glabrata at different developmental stages. PLos Negl Trop Dis 7(6): 2251.

6. Retief E (2000) Lamiaceae (Labiatae). In: Leistner OA (Ed.), Seed plants of Southern Africa: Strelitzia. 10: 323-334.

7. Rice LJ, Brits GJ, Potgieter CJ, Van Staden J (2011) Plectranthus: A plant for the future? South African Journal of Botany 77(4): 947-959.

8. Paraense WL, Corrêa LR (1988) Self-fertilization in the freshwater snails Helisoma dury and Helisoma trivolvis. Mem Inst Oswaldo Cruz 83: 405409 .

9. Kawai M, Matsuura T, Nakeshima R, Batsugan YJ (1970) Structures of physalin A and physalin B, 13,14-seco-16,24-cyclo-steroids from Physalis alkekengi var. Francheti. Chem Soc C 5: 664-670.

10. Makino B, Kawai M, Iwata Y, Yamamura H, Batsugan Y, et al. (1995) Physalins possessing an endoperoxy structure from Physalis alkekengi var. francheti. Structural Revision of Physalin K. Bull Chem Soc Jpn 68: 219.

11. Santos JAA, Cavalcante VP, Rangel LS, Leite JCVA, Faria RX (2017) A new technique using low volumes: Anew technique to assess the molluscicidal activity using low volumes. Evidence-Based Complementary and Alternative Medicine 2017: 10

12. McCullough FS (1980) Molluscicides in schistosomiasis control. Bulletin of the World Health Organization 58(5): 681-689.

13. Pereira Filho AA, Franca CR, Oliveira S, Mendes RJ, Gonçalves R, et al. (2014) Evaluation of the molluscicidal potential of hydroalcoholic extracts of Jatropha gossypiifolia Linnaeus, 1753 on Biomphalaria glabrata (Say, 1818). Rev Inst Med Trop 56(6): 505-510.

14. Bandeira JM, Barbosa FF, Barbosa LMP, Rodrigues ICS, Bacarin MA, et al. (2011) Essential oil composition of four species of the genus Plectranthus. 13(2): 157-164.

15. Costa AV, Almeida BR, Gonçalves LV, Crico KB, Ignacchiti MDC, et al. (2015) Efeito moluscicida do óleo essencial de Cymbopogon winterianus Jowitt (Poaceae) sobre Lymnaea columella (Say, 1817) e Biomphalaria tenagophila (D’Orbigny, 1835). Rev Bras Pl Med Campinas 17(4): 707712.

16. Santos JAA, Tomassini TCB, Xavier DCD, Ribeiro IM, Silva MTG, et al. (2003) Molluscicidal activity of Physalis angulata L. extracts and fractions on Biomphalaria tenagophila (d'Orbigny, 1835) under laboratory conditions. Mem Inst Oswaldo Cruz, Rio de Janeiro 98(3): 425-428.

17. Otarigho B, Morenikeji OA (2012) Molluscicidal effects of aqueous and ethanolic extracts of lemongrass (Cymbopogon citratus) leaf against the different developmental stages of Biomphalaria pfeifferi. New York Science Journal 5(8).

18. Araújo FP, Albuquerque RDDG, Rangel LS, Caldas GR, Tietbohl LAC, et al. (2019) Nanoemulsion containing essential oil from Xylopia ochrantha Mart. produces molluscicidal effects against different species of Biomphalaria (Schistosoma hosts). Mem Inst Oswaldo Cruz, Rio de Janeiro 114: e180489. 